\title{
EFFECT OF OBSERVING DIFFERENT MODEL DEMONSTRATIONS ON THE DEVELOPMENT OF INTERNAL MOTOR REPRESENTATION
}

\author{
Ghorbani Saeed ${ }^{1 *}$ \\ Schuster Frerk ${ }^{2}$ \\ Hillebrecht Martin ${ }^{3}$ \\ Bund Andreas ${ }^{4}$ \\ ${ }^{1}$ Department of Physical Education and Sport Science, Aliabad Katoul Branch, Islamic \\ ${ }^{2}$ Azad University, Aliabad Katoul, Iran \\ ${ }^{3}$ Institute of Sport Science, University of Oldenburg, Germany \\ ${ }^{4}$ Institute of Applied Educational Sciences, University of Luxembourg, Luxembourg
}

Keywords: Observation, Error-detection, Baseball-pitch

\begin{abstract}
This study was designed to investigate the relative effects of observing video, pointlight, and stick-figure model demonstrations on the development of internal motor representation of a highly complex sport skill. Forty one novice female and male students were randomly assigned to video, point-light, stick-figure and nodemonstration control groups. Internal motor representation was evaluated by a computer - based test using the error detection paradigm. Participants had to view ten digital photos representing different phases of a Baseball pitching and were instructed to identify by mouse-clicking various movement errors. The test was respectively performed after 5 familiarization trials (pre-test), 3 acquisition blocks of 10 trials (posttest) and one week without practice (retention test). Participants observed related model demonstrations prior to each acquisition block. Results showed that demonstration groups improved their scores in either post-test or retention test; however these improvements were not statistically significant. Moreover, there was no significant difference between groups either in post-test or retention test. The findings are discussed in terms of difficulty of errors, insufficient amount of physical or observational practice, and small sample size.
\end{abstract}

\section{Introduction}

Learning a new motor skill is primarily a process by which learners have to acquire a new spatiotemporal coordination pattern. Model demonstration is commonly used by instructors and coaches as an instructional strategy to facilitate acquisition of movement coordination pattern in sport setting. A meta-analysis of the literature on observational motor learning revealed that observing a model has

*E-mail: s.ghorbani@aliabadiau.ac.ir 
a strong effect (0.77) on movement dynamics and a small effect (0.17) on movement outcome in a wide range of motor skills (Ashford et al. 2006).

According to Bandura's (1986) social cognitive theory, observing a model results in the development of a "cognitive representation". As such, the extracted information are mentally rehearsed by the observers and retained in the form of a symbolic representation which later serves as an internal model to guide the action reproduction and error detection-correction mechanism. To provide support for Bandura`s theory, a number of studies have investigated the development of cognitive representation through observing a model (e.g., Black \& Wright, 2000; Black et al. 2005; Blandin \& Proteau, 2000; Carroll \& Bandura, 1982, 1985, 1987, 1990). A series of experiments conducted by Carroll and Bandura have demonstrated that action observation leads to the set of a standard model for movement recognition and production (Carroll \& Bandura, 1982, 1985, 1987, 1990). Moreover, by using a causal approach Carroll and Bandura, 1990 confirmed that the development of an internal cognitive representation mediates the later reproduction of action and a mechanism for detection-correction of errors. In addition, Black et al. (2005), and Blandin and Proteau, (2000) reported that participants who observed a model were able to estimate the timing of their movement as accurate as those who physically performed the task. As a result, Blandin and Proteau, (2000), proposed that physical and observational practice engages the individuals in similar cognitive processes.

This proposition is also consistent with the findings of neurophysiological and brain imaging studies indicating that a particular class of visuomotor neurons, called "Mirror Neurons", exist in human brain and that discharge both during execution of an action and also observing the same action performed by others (Rizzolatti, 2005; Rizzolatti \& Craighero, 2004). Moreover, it has been shown that the mirror neuron system provides an important neural substrate that supports the acquisition of new movement patterns (Buccino et al. 2004). The authors pointed out that mirror neurons represent the neurological basis of imitation learning by translating the elementary motor acts of observed actions into the motor representation of the same action in prefrontal cortex, ventral premotor cortex and the pars opercularis of inferior frontal gyrus, and by selecting and recombining motor elements in area 46.

Recently, in addition to video display, researchers applied digital processed animations of sport skills such as point-light or stick-figure displays into observational learning research. Theoretical basis for applying point-light or stick-figure displays is originated from the Visual Perception Perspective (Scully \& Newell, 1985). This theory suggest that while observing a demonstration, relative motion information of the action, i.e., the spatiotemporal changes of body joints or extremities in relation to each other, is directly extracted and perceived by the observer and later used to reproduce the modeled motor skill. According to this theory, a model demonstration should be particularly effective when relative motion information of the movement is 
highlighted. That can be achieved by representing the human body in the form of a point-light or stick-figure display rather showing the observers a classic video containing structural information. If so, observing a point-light or a stickfigure demonstration should result in better motor performance and learning than observing a classic video demonstration.

Scully and Carnegie (1998) showed that observing a point-light display was more effective in acquisition of a gymnastic dance skill in terms of movement coordination in comparison to a video display. Moreover, Horn et al. (2005), Breslin et al. (2005) and Rodrigues et al. (2010) found that participants in a point-light demonstration group were capable to replicate the movement form of a soccer kicking, bowling cricket, and ballet dance actions, respectively, as similar as participants in a video demonstration group.

While there is now considerable experimental evidence that observing point-light or stick-figure demonstrations facilitate the acquisition of new movement patterns, it must be stated that no studies exist that examine the development of internal motor representation through observing such processed displays. Therefore, the present study was designed to investigate the effects of observing point-light, stick-figure and video displays on the development of internal motor representation of a complex and multi-limbs sport skill.

Internal motor representation was measured by means of computer software developed by one of the co-authors of this paper. Using an errordetection paradigm, participants were presented a set of ten photos of a male pitcher including significant movement errors at different phases of the pitch. Participants were then asked to click with a computer mouse on that part of the body (e.g. right or left arm, foot, etc.) which they believe to be performed incorrectly.

During experimental procedure, the test was applied three times including after five familiarization trials (pretest), after three acquisition blocks of ten trials (posttest), and about one week later (retention test). Before each acquisition block participants in each experimental group observed related model demonstrations three times on a laptop screen. Participants in the control group followed the same regime but without observing a model demonstration.

Based on the results of previous research (Breslin et al., 2005; Horn et al., 2005; Rodrigues et al., 2010; Scully \& Carnegie, 1998) it was hypothesized that point-light and stick-figure displays are at least as effective as a video display for developing internal motor representation. It was also expected that participants who observe point-light, stick-figure and video demonstrations would develop internal motor representation better than those who observed no model demonstration.

\section{Material and methods}

Forty one male and female students of the University of Oldenburg (mean age $=24.2$ years old) participated voluntarily in the study. All were right side dominant and naive to the criterion task. Participants were randomly assigned to 
one of four groups including video, point-light, stick-figure and no demonstration control groups. All groups had equal number of males and females with exception of video group with 6 females. The study was performed in accordance with the Ethical Standards laid down in the Deceleration of Helsinki. All participants gave informed written consent.

A highly complex and dynamic throwing action, the Baseball-pitch, was selected as criterion task. Due to his clear phase structure and because it is easy to determine significant movement errors for each phase, the Baseball-pitch was considered as a suitable motor task for this study. A right-handed male pitcher (age $=32$ years) with seven years playing experience in the second Baseball league of Germany acted as video model. First, a classic video was produced by recording the execution of a Baseball-pitch performed by the expert with a digital video camera from a sagittal plane (Figure 1a).

To produce point-light and stick-figure videos, retro-reflective markers were then attached to the forehead, shoulder, elbow, wrist, hip, knee, ankle, and toe joints on left and right side of his body. Four digital and synchronized cameras filmed spatiotemporal positions of markers while he performed various pitches. Simi Motion software 5.0 ${ }^{\mathrm{TM}}$ (SIMI Reality Motion Systems GmbH, Germany) was used to compile point-light and stick-figure videos. Point-light video was generated by processing the recording so that only the markers were visible as point-lights in a darkened background (Figure 1b).

Stick-figure video was composed of the similar light points connected to each other with lines (Figure 1c).

All video demonstrations involved identical start and end points with four seconds duration.
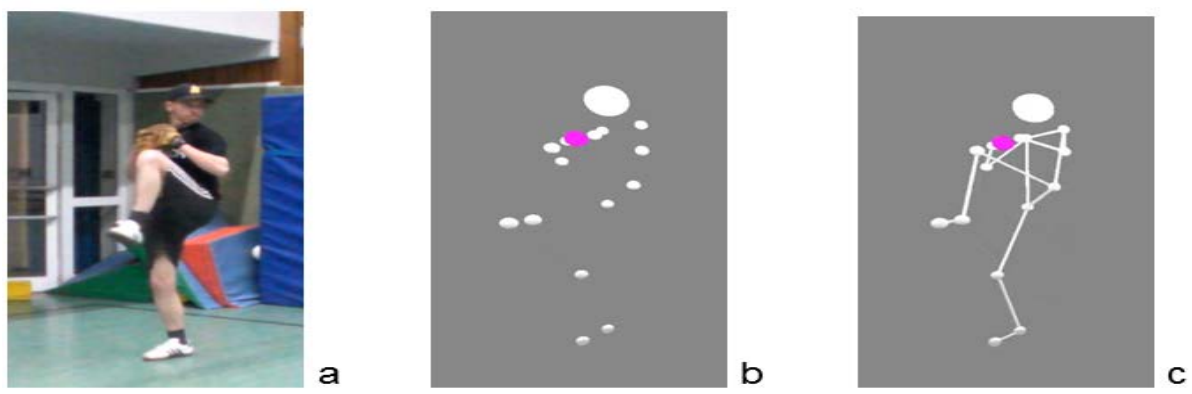

Figure 1. Screenshots of video demonstrations used in the study. a) Video, b) Point-light, c) Stick-figure.

\section{Internal motor representation}

An error detection paradigm was applied to evaluate the development of internal motor representations through observation and reproduction of action. We divided the pitching skill into three phases including preparation (windup, stride), main (arm cocking, arm acceleration) and end (arm deceleration, followthrough) phases. 
An initial pool of sixteen photos showing the execution of a Baseball-pitch by a male person was created with the collaboration of an experienced Baseballpitch. Each photo represented a typical movement error at a certain time point during pitching. Six photos of this pool were removed after an expert review due to their similarity with some other photos.

The final set of 10 photos was standardized using a photo editing software (Paint), so that the background of each photo was colored in white, the person was located in the center, and resolution was $1024 \times 728$ pixels. The photos were then integrated into a self-developed software program called ErrorDetection-Test (ETD).

The movement errors were: 1 . Striding leg is not elevated; 2 . Throwing arm is stretched; 3. Supporting leg is elevated; 4. Legs are flexed; 5 . Backswing of the throwing arm is too short; 6- Big step during preparation phase; 7. Back leg stays behind; 8. Ball is released too late; 9 . Feet of striding leg do not point in throwing direction during main phase; and 10. Opposite arm (catcher's mitt) don't cross the central axis of body during end phase.

An example of movement errors presented in EDT is shown in Fig. 2.

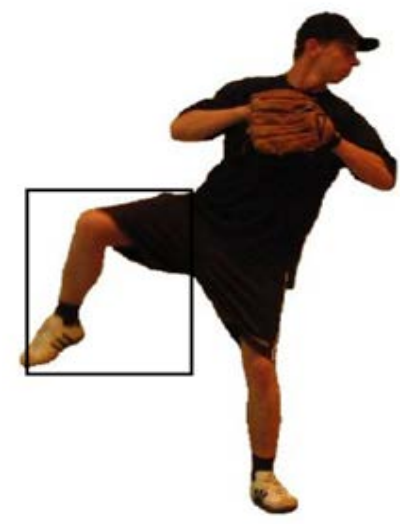

Figure 2. Photo number 3 in the ETD which shows the movement error „supporting leg is elevated“.

The ETD-software was programmed in a way that the movement error on each photo was assigned to a spatial corresponding rectangular "error area". Within the software, we measured a variable called "number of hits" as dependent variable. Number of hits refers to successful error detections, in which a mouse click on a point inside the error area was regarded as correct error detection (1 point) and a click outside this area as wrong error detection (0 point). As such, number of hits score is ranged from 0 to 10 . Before beginning the EDT, participants were given an introduction to the software followed by an example (one of the six removed photos). Photos then appeared in random order and were visible in each case for ten seconds. On each photo the mouse pointer was automatically centered at the beginning. 
According to a consensus decision of coach and investigators, first five of the presented movement errors were classified as easily to find, the second five errors as difficult.

\section{Procedure}

Participants were tested individually in two days. On the first day and after an introduction to the protocol participants were given instruction of the Baseball-pitch consisted of a series of images of pitch phases supplemented by extra notions of main features of the phases. After 5 familiarization trials the participants received the pretest. Following completion of the pretest participants performed three blocks of 10 trials as acquisition phase of the experiment.

Participants observed video demonstrations related to their experimental group displayed on a laptop screen three times before each acquisition block. Participants of control group followed the same protocol, but did not observe model demonstration. Posttest and retention test were performed 10 min after acquisition phase and one week later, respectively, without providing further practice or demonstrations.

The number of hits score was analyzed by using a mixed analysis of variance (ANOVA) in which experimental group (GROUP) was the betweensubject factor and the time of test (TIME) was the within-subjects factor. The significant level for all statistical analyses in this study was set at $p<0.05$. Additionally, the partial eta ${ }^{2}$ was calculated as effect size.

\section{Results and Discussions}

Table 1 and Figure 3 show the means and standard deviations of number of hits, i.e., number of times participants hit correctly inside the area error. In the pretest, the highest average values were achieved in the video and the control group with 3.91 and 3.90, point light group performed worse with 3.4. In the posttest, the values of the point-light and control group remained approximately the same, while the number of hits on video and stick-figure group increased to 4.45 and 4.10, respectively. Compared to the previous test, in the retention test the values of the control group remained relatively stable.

The video group went in their hit rate back to 3.91, the same value that was achieved in the pretest. The stick-figure group achieved same number of hits as posttest. The point-light Group increased its average of 0.7 up to 4.00. The ANOVA on scores of this variable revealed no significant main effect for GROUP $\left(F_{3,37}=0.74, p=0.47, \varepsilon_{p a r}{ }^{2}=0.02\right)$, TIME $\left(F_{2,74}=0.61, p=0.72, \varepsilon p a r^{2}\right.$ $=0.04)$, or INTERACTION $\left(F_{6,74}=0.47, p=0.69, \varepsilon_{p a r}{ }^{2}=0.03\right)$.

The results of previous research have demonstrated that point-light display is at least as effective as video display for learning a new motor skill (Horn et al. 2005; Breslin et al. 2005). Also it has been shown that action observation results in detecting movement timing errors as similar as physical practice (Black \& Wright, 2000; Black et al. 2005; Blandin \& Proteau, 2000). The primary aim of this experiment was to extent these results by comparing the 
effects of observing point-light, stick-figure and video displays on development of internal motor representations of a complex sport skill.

Based on the previous research, it was hypothesized that point-light, stickfigure and video demonstration groups would not differ from each other in terms of error-detection scores during both post- and retention tests, but these groups were hypothesized to achieve better scores in comparison to no-demonstration control group.

Table 1 Means and standard deviations of number of hits across groups and time of test

\begin{tabular}{cccc}
\hline Groups & Pretest & Posttest & Retention test \\
Video & $3.91 \pm 0.70$ & $4.45 \pm 1.44$ & $3.91 \pm 1.38$ \\
Point-light & $3.40 \pm 1.71$ & $3.30 \pm 1.16$ & $4.00 \pm 1.41$ \\
Stick-figure & $3.60 \pm 0.41$ & $4.10 \pm 0.45$ & $4.10 \pm 0.47$ \\
Control & $3.90 \pm 1.29$ & $4.00 \pm 1.41$ & $3.90 \pm 1.60$ \\
\hline
\end{tabular}

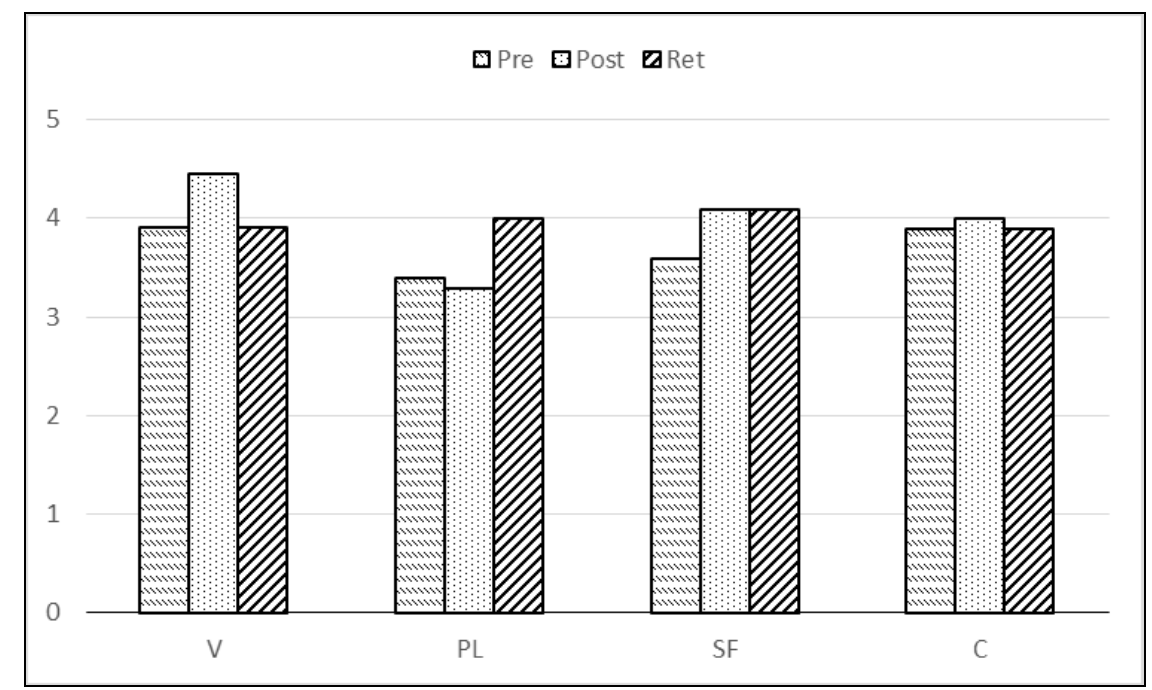

Figure 3. Means of number of hits across groups and time of test

Results of the study showed that there was no significant difference between the four groups in terms of the number of hits either in post- or retention test. This finding, therefore, did not provide support for the our hypothesis and, therefore, the notion that observing a video, stick-figure or point-light demonstration is more effective for developing internal motor representations than no-model demonstration.

However, the lack of statistically significant differences for the demonstration groups does not necessarily mean that demonstration of the to-belearnt skill is of no use for the development of an internal representation of the 
motor skill. A thorough examination of the scores of all groups (means and standard deviations) apparently indicated that the participants who were observing a video, stick-figure or point-light model improved their scores somewhat more but non-significantly during the posttest and retention test, whilst the participants who did not observe a model showed no improvement in their scores either in post or retention test. Nonetheless, it must be stated that these findings are not in line with previous research which found that model observation affects development of an error-detection mechanism (Black and Wright, 2000; Black et al. 2005; Blandin and Proteau, 2000) as well as cognitive representations (Carroll and Bandura, 1982, 1985, 1987, 1990).

In our opinion, there could be three plausible explanations for these findings. The first one is that the lack of a benefit of observing a model may have been due to the difficulty of errors presented in EDT. Previous research has often employed an error recognition paradigm including movement time estimation (Black and Wright, 2000; Black, et al. 2005; Blandin and Proteau, 2000) or arranging the photos of movement sequences in a correct spatiotemporal order (Carroll and Bandura, 1982, 1985, 1987, 1990).

In the present study, a multi-limb and highly complex sport skill was used as criterion task. The relatively low scores of error-detection for participants in all groups confirm the statement that the movement errors shown on the photos had a level of difficulty, that the majority of participants were not able to recognize them. The average score of participants across all times of measurement were less than a half of photos. The best single score throughout this experiment was attained by a participant in the video group who was able to recognize 7 of 10 errors in post test. Altogether, it seems possible that participants had somewhat difficult to detect errors even after observing the model and performing the motor task physically.

Second, due to the relative complexity of the task the amount of practice and exposure to the model was insufficient to cause relevant improvements in EDT-scores across all measures. This might be another reason for relatively low overall mean scores for the groups. Given that the participants in the present study only performed $3 \times 10$ trials during acquisition phase and observed $3 \times 3$ times the respective model, a best error detection performance might be achieved through increasing the number of physical and observational interactions with the motor task.

Finally, it could be possible that sample size used in this study was not sufficient to increase the probability of obtaining a significant difference between groups if it is present. We used 11 participants in video groups and 10 participants in point-light, stick-figure and control groups. Increasing number of the participants is often the easiest method to boost the statistical power of a test. It might be possible that increasing the number of participants in each experimental group obtain a significant difference between groups or increase the possibility of producing an improvement during the time of the test. 
In summary, the results of this study revealed that observation of a model does not facilitate in any case the development of motor representation in terms of error-detection competencies. However, some non-significant improvements were observed in post- or retention test in video, stick-figure or point-light groups. The lack of significant differences may be due to the difficulty of errors presented in EDT, low amount of physical practice and exposure to model, and small sample size. Further research is needed to examine the effectiveness of observing a model (e.g., point-light, stick-figure or video) on development of internal motor representation of a complex motor skill - also by using other approaches to measure the structure and quality of internal motor representation.

\section{References}

1. ASHFORD, D., BENNETT, S.J., DAVIDS, K. (2006). Observational modeling effects for movement dynamics and movement outcomes across differing task constraints: A meta-analysis. Journal of Motor Behavior, 38(3): 185-205.

2. BANDURA, A. (1986). Social foundations of thought and action: A social cognitive theory. Englewood Cliffs, NJ: Prentice-Hall.

3. BLACK, C.B., WRIGHT, D.L. (2000). Can observational practice facilitate error recognition and movement production? Research Quarterly for Exercise and Sport, 71(4): 331-339.

4. BLACK, C.B., WRIGHT, D.L., MAGNUSON, C.E., BRUECKNER, S. (2005). Learning to detect error in movement riming using physical and observational practice. Research Quarterly for Exercise and Sport, 76(1): 28-41.

5. BLANDIN, Y., PPORTEAU, L. (2000). On the cognitive basis of observational learning: Development of mechanisms for detection and correction of errors. The Quarterly Journal of Experimental Psychology, 53A(3): 846-867.

6. BRESLIN, G., HODGES, N.J., WILLIAMS, M.A., CURRAN, W., KREMER, J. (2005). Modeling relative motion to facilitate intra-limb coordination. Human Movement Science, 24: 446-463.

7. BUCCINO, G., VOGT, S., RITZL, A., GEREON, R., FINK, G.R., ZILLES, K., FREUND, H., RIZZOLATTI, G. (2004). Neural circuits underlying imitation learning of hand actions: An event-related fMRI study. Neuron, 42: 323-334.

8. CARROLL, W.R., BANDURA, A. (1982). The role of visual monitoring in observational learning of action patterns: Making the unobservable observable. Journal of Motor Behavior, 14: 153-167.

9. CARROLL, W.R., BANDURA, A. (1985). Role of timing of visual monitoring and motor rehearsal in observational learning of action patterns. Journal of Motor Behavior, 17: 269-281. 
10. CARROLL, W.R., BANDURA, A. (1987). Translating cognition into action: The role of visual guidance in observational learning. Journal of Motor Behavior, 19: 385-398.

11. CARROLL, W.R., BANDURA, A. (1990). Representational guidance of action production in observational learning: A causal analysis. Journal of Motor Behavior, 22: 85-97.

12. FELTZ, D.L. (1982). The effects of age and number of demonstrations on modeling of form and performance. Research Quartely for Exercise and Sport, 53(4): 291-296.

13. HORN, R.R., WILLIAMS, M.A., SCOTT, M.A., HODGES, N.J. (2005). Visual search and coordination changes in response to video and point-light demonstrations without KR. Journal of Motor Behavior, 37(4): 265-274.

14. LANDERS, D.M., LANDERS, D.M. (1973). Teacher versus peer models: Effects of model's presence and performance level on motor behavior. Journal of Motor Behavior, 5 (3): 129-139.

15. RIZZOLATTI, G. (2005). The mirror neuron system and its function in humans. Anatomy and Embryology, 210: 419-421. DOI 10.1007/s00429005-0039-z.

16. RIZZOLATTI, G CRAIGHERO, L. (2004). The mirror-neuron system. Annual Review of Neuroscience, 27:169-92. doi: 10.1146/annurev.neuro.27.070203.144230.

17. RODRIGUES, S.T., FERRACIOLI, M.C., DENARDI, R.A. (2010). Learning a complex motor skill from video and point-light demonstrations. Perceptual and Motor Skills, 111(1): 1-17.

18. SCULLY, D.M., NEWELL, K.M. (1985). The Acquisition of Motor Skills: Toward a Visual Perception Perspective. Journal of Human Movement Studies, 12: 169-187.

19. SIDAWAY, B., HAND, J.M. (1993). Frequency of modeling effects on the acquisition and retention of a motor skill. Research Quarterly for Exercise and Sport, 64(1): 122-126.

20. WEIR, P.L., LEAVITT, J.L. (1990). Effects of model`s skill level and model `s knowledge of results on the performance of a dart throwing task. Human Movement Science, 9: 369-383. 